\title{
Autonomous Aerial Refueling Modeling and Dynamic Inversion Adaptive Sliding Mode Control
}

\author{
Sun Xun \\ Flight Control Division \\ Xi'an Flight Automatic Control Research Institute \\ Xi’an, P.R.China \\ nwpusunxun@sina.com
}

\begin{abstract}
A dynamic inversion adaptive sliding mode flight control method for autonomous aerial refueling (AAR) is approved in this paper. The drogue model and refueling aircraft 6-DOF nonlinear model is built. In order to improve the flight control precision and performance, the dynamic inversion control method is used to design the flight control law. Fuzzy adaptive control is combined with the sliding mode control is used to compensate the error of inversion, so the robustness and transient characteristic performance could be improved. The simulation results show that the AAR flight control has excellent control performance.
\end{abstract}

Keywords-autonomous aerial refueling; dynamic inversion; sliding mode control; drogue model

\section{INTRODUCTION}

UAV is becoming more and more important military and civil airplane. In the help of the UAV, more complex and more dangerous mission could be accomplished. Autonomous aerial refueling technique is a critical capability for the UAV. The AAR could solve the contradiction between the complex mission requirement and fuel limit. The autonomous aerial refueling is a effective method to improve the range of the UAV. For long flight time UAV, one aerial refueling could improve the flight time more than $80 \%$. For combat UAV, one aerial refueling could improve the flight time more than $30 \%^{[3-6]}$.

The AAR could be divided into four phases: closure, capture, hold and unplug. For each phase, the high precision and stability control is the key technique. Because of the disturbance coming from the gust, drogue movement, tanker airplane movement, refueling movement and quality changing, it is difficult to realize the AAR high precision control.

In order to improve the control performance in AAR flight phase, a dynamic inversion adaptive sliding mode flight control method is designed. In this AAR flight controller, dynamic inversion method is used to realize the nonlinear control and fuzzy adaptive module is used to compensate the error of inversion. For improving the robustness, sliding mode control is used to optimize the adaptive control.

\author{
Zhang Xinguo \\ Flight Control Division \\ Xi'an Flight Automatic Control Research Institute \\ Xi'an, P.R.China \\ fcd@facri.com
}

\section{Autonomous Refueling Modeling}

\section{A. Drogue Model}

The diameter of the drogue is 32 inch. Its dynamic model in terms of the stiffness and damp coefficient is built as follow ${ }^{[2]}$.

$$
\begin{gathered}
\dot{x}_{d}(t)=A_{d} x_{d}(t)+B_{d} w(t) \\
\dot{y}_{d}(t)=C_{d} x_{d}(t)
\end{gathered}
$$

Where: $x_{d}=\left[\begin{array}{llllll}\Delta x_{d} & \Delta y_{d} & \Delta z_{d} & \Delta \dot{x}_{d} & \Delta \dot{y}_{d} & \Delta \dot{z}_{d}\end{array}\right]$.

The matrix was selected as:

$$
\begin{gathered}
A_{d}=\left[\begin{array}{cccccc}
0 & 0 & 0 & 1 & 0 & 0 \\
0 & 0 & 0 & 0 & 1 & 0 \\
0 & 0 & 0 & 0 & 0 & 1 \\
-k_{x} & 0 & 0 & -c_{x} & 0 & 0 \\
0 & -k_{y} & 0 & 0 & -c_{y} & 0 \\
0 & 0 & -k_{z} & 0 & 0 & -C_{z}
\end{array}\right] \\
B_{d}=\left[\begin{array}{cccc}
0 & 0 & 0 \\
0 & 0 & 0 \\
0 & 0 & 0 \\
0.01 & 0 & 0 \\
0 & 0.3 & 0 \\
0 & 0 & 0.3
\end{array}\right] \\
C_{d}=\left[\begin{array}{cccccc}
1 & 0 & 0 & 0 & 0 & 0 \\
0 & 1 & 0 & 0 & 0 & 0 \\
0 & 0 & 1 & 0 & 0 & 0
\end{array}\right]
\end{gathered}
$$

The values of the $k_{x}, k_{y}, k_{z}, c_{x}, c_{y}, c_{z}$ are $0.15,0.06$, $0.1,0.5,0.04,0.05$.

\section{B. Refueling Aircraft Modeling}

The 6-DOF nonlinear airplane model in the body axis are: 
$\frac{\mathrm{d} v}{\mathrm{~d} t}=\frac{P}{m}-\frac{Q}{m} \cos \alpha \cos \beta+\frac{Y}{m} \sin \alpha-g \sin \vartheta$

$\frac{\mathrm{d} \alpha}{\mathrm{d} t}=\omega_{z}-\beta \omega_{x}-\frac{Y}{m V} \cos \alpha-\frac{Q}{m V} \sin \alpha+\frac{g}{V} \cos \vartheta \cos \gamma$

$\frac{\mathrm{d} \beta}{\mathrm{d} t}=\omega_{y}+\alpha \omega_{x}+Z+\frac{g}{V} \cos \vartheta \sin \gamma$

$$
\frac{\mathrm{d} \omega_{x}}{\mathrm{~d} t}=B_{y} \omega_{y} \omega_{z}-B_{x y} \omega_{x} \omega_{y}+M_{x}
$$

$$
\frac{\mathrm{d} \omega_{y}}{\mathrm{~d} t}=B_{x y} \omega_{y} \omega_{z}-B_{x} \omega_{x} \omega_{z}+M_{y}
$$

(10)

$$
\frac{\mathrm{d} \omega_{z}}{\mathrm{~d} t}=\frac{J_{x}-J_{y}}{J_{z}} \omega_{x} \omega_{y}-\frac{J_{x y}}{J_{z}}\left(\omega_{x}^{2}-\omega_{y}^{2}\right)+M_{z}
$$

(11)

$$
\frac{\mathrm{d} \gamma}{\mathrm{d} t}=\omega_{x}-\operatorname{tg} \vartheta\left(\cos \gamma \omega_{y}-\omega_{z} \sin \gamma\right)
$$

(12)

$$
\begin{aligned}
\frac{\mathrm{d} \vartheta}{\mathrm{d} t} & =\omega_{y} \sin \gamma+\omega_{z} \cos \gamma \\
\frac{\mathrm{d} \varphi}{\mathrm{d} t} & =\frac{1}{\cos \vartheta}\left(\omega_{y} \cos \gamma-\omega_{z} \sin \gamma\right) \\
\frac{\mathrm{d} h}{\mathrm{~d} t} & =V \sin (\vartheta-\alpha) \\
\frac{\mathrm{d} z}{\mathrm{~d} t} & =-V \sin (\varphi-\beta) \\
\frac{\mathrm{d} L}{\mathrm{~d} t} & =V \cos (\vartheta-\alpha)
\end{aligned}
$$

Where: $V, m, P, Q, Y, Z, \alpha, \beta, \vartheta, \gamma, \varphi, \omega_{x}, \omega_{y}$, $\omega_{z}, M_{x}, M_{y}, M_{z}, h, z, L$ are velocity, mass, thrust, drag, lift, lateral force, angle of attack, angle of sideslip, pitch angle, roll angle, yaw angle, rate of roll angle, rate of yaw angle, rate of pitch angle, roll moment, yaw moment, pitch moment, flight altitude, lateral distance, flight distance.

\section{LARGE ENVELOPE NEURAL NETWORK ADAPTIVE FUZZY SLIDING Mode Flight CONTROLlER}

\section{A. Dynamic Inversion Control}

The system is:

$$
\dot{x}=f(x)+g(x) u
$$

If $g(x)$ could been inverted, then:

$$
u=g^{-1}(x)\left[w_{c}\left(x_{c}-x\right)-f(x)\right]
$$

Where: $w_{c}$ is bandwidth, $x_{c}$ is command input.
The out character of the system is:

$$
\dot{x}=w_{c}\left(x_{c}-x\right)
$$

It is obviously that the system is stability.

Take angle rate as the inner loop output, the inner loop equations are:

$$
\left[\begin{array}{c}
\dot{w}_{x} \\
\dot{w}_{z} \\
\dot{w}_{y}
\end{array}\right]=\left[\begin{array}{c}
f_{w x}(x) \\
f_{w z}(x) \\
f_{w y}(x)
\end{array}\right]+g(x)\left[\begin{array}{c}
M x \\
M y \\
M z
\end{array}\right]
$$

In some situation, it could been expressed as this form.

$$
\left[\begin{array}{c}
\dot{w}_{x} \\
\dot{w}_{z} \\
\dot{w}_{y}
\end{array}\right]=\left[\begin{array}{c}
f_{w x}(x) \\
f_{w z}(x) \\
f_{w y}(x)
\end{array}\right]+g(x) \delta
$$

For the outer loop, the command form is $\left(\alpha_{c}, \beta_{c}, \gamma_{c}\right)$. The outer loop state equation could been expressed as:

$$
\left[\begin{array}{c}
\dot{\alpha} \\
\dot{\beta} \\
\dot{\gamma}
\end{array}\right]=\left[\begin{array}{l}
f_{\alpha}(x) \\
f_{\beta}(x) \\
f_{\gamma}(x)
\end{array}\right]+g(x)\left[\begin{array}{l}
w_{x} \\
w_{z} \\
w_{y}
\end{array}\right]
$$

The angle rate command is expressed as:

$$
\left[\begin{array}{c}
w_{x c} \\
w_{z c} \\
w_{y c}
\end{array}\right]=g^{-1}(x)\left(\left[\begin{array}{c}
\dot{\alpha}_{d} \\
\dot{\beta}_{d} \\
\dot{\gamma}_{d}
\end{array}\right]-\left[\begin{array}{c}
f_{\alpha}(x) \\
f_{\beta}(x) \\
f_{\gamma}(x)
\end{array}\right]\right)
$$

\section{B. Adaptive Control Based on Fuzzy System}

Because of the uncertainty and the complexity of the model, the motion equation is hard to precise description and generate the error of inversion $\Delta(x, \dot{x}, u)=f(x, \dot{x}, u)-\hat{f}(x, \dot{x}, u)$. The inversion error would generate the severe influence on the system stabilization. The fuzzy adaptive module is used to counteract the influence of the inversion error.

Select the GAUSS membership function, the adaptive output is:

$$
U_{j}^{a d}=\frac{\sum_{L=1}^{M} \bar{y}_{j}\left[\prod_{i=1}^{n} a_{i}^{L} \exp \left(-\left(\frac{x_{i j}-\bar{x}_{i j}^{L}}{\sigma_{i j}^{L}}\right)^{2}\right)\right]}{\sum_{L=1}^{M}\left[\prod_{i=1}^{n} a_{i}^{L} \exp \left(-\left(\frac{X_{i j}-\bar{x}_{i j}^{L}}{\sigma_{i j}^{L}}\right)^{2}\right)\right]}
$$

In order to speed up the convergence rate, suppose the $\bar{x}_{i j}^{L}, \sigma_{i j}^{L}, a_{i}^{L}$ are constant

$$
U_{j}^{a d}=W_{j} Z(x)
$$

In the effect of pseudo control parameter $U=U^{0}-U^{a d}$, the tracking error dynamic equation is: 


$$
\dot{\tilde{Y}}_{j}=-A_{j} \tilde{Y}_{j}+B\left(\Delta_{j}-U_{j}^{a d}\right)
$$

Select the Lyapunov function:

$$
V_{j}=\left\{\begin{array}{l}
\frac{1}{2} \tilde{Y}_{j}^{T} \tilde{Y}_{j}+\frac{1}{2 k_{j}} \tilde{W}_{j}^{T} \tilde{W}_{j},\left\|\tilde{Y}_{j}\right\|>E_{j} \\
\frac{1}{2} E_{j}^{2}+\frac{1}{2 k_{j}} \tilde{W}_{j}^{T} \tilde{W}_{j},\left\|\tilde{Y}_{j}\right\| \leq E_{j}
\end{array}\right.
$$

(28)

1) When $\left\|\tilde{Y}_{j}\right\| \leq E_{j}$, the time derivation Lyapunov function $\dot{V}_{j}=0$;

2) When $\left\|\tilde{Y}_{j}\right\|>E_{j}$,

$\dot{V}_{j}=\tilde{Y}_{j}^{T} \dot{\tilde{Y}}_{j}+\frac{1}{k_{j}} \tilde{W}_{j}^{T} \dot{W}_{j}=-\tilde{Y}^{T} A_{j} \tilde{Y}_{j}+\tilde{Y}_{j}^{\tau} B \varepsilon_{j} \leq-\lambda_{\min }\left(A_{j}\right)\left\|\tilde{Y}_{j}\right\|^{2}+\left|\varepsilon_{j}\right| \| \tilde{Y}_{j}||<0$

\section{The Robustness Compensate Using Sliding Mode Control}

Sliding mode control has strong robustness for un-model dynamics and environment disturbance, so using sliding mode control to improve the robustness and control performance.

Sliding surface $s_{j}\left(\tilde{y}_{j}, t\right)=\tilde{y}_{j}=0$, suppose $\dot{s}_{j}=0$, equivalent pseudo control is $\hat{U}_{j}^{s}=\hat{\dot{y}}_{j}$ 。

The robust compensation is $U_{j}^{s}=\rho_{j} \operatorname{sgn}\left(s_{j}\right)$, then

$$
\dot{s}_{j}=\Delta_{j}-U_{j}^{a d}-U_{j}^{s}-g \dot{s}_{j}
$$

Suppose $\tilde{\rho}_{j}=\rho_{j}-\rho_{j}^{*}, \quad \rho_{j}^{*}$ is residual,

(30)

$$
\left\{\begin{array}{c}
\dot{\tilde{W}}_{j}=\dot{W}_{j}=\lambda_{j} s_{j} Z(x) \\
\dot{\tilde{\rho}}_{j}=\dot{\rho}_{j}=\eta_{j}\left|s_{j}\right|
\end{array}\right.
$$

Select the Lyapunov function

$$
V_{j}=\frac{1}{2} s_{j}^{2}+\frac{1}{2 \lambda_{j}} \tilde{W}_{j}^{T} \tilde{W}_{j}+\frac{1}{2 \eta_{j}} \tilde{\rho}_{j}^{2}
$$

Then, $\dot{V}_{j}<-g s_{j}^{2}+\rho_{j}^{*}\left|s_{j}\right|-\rho_{j}\left|s_{j}\right|+\tilde{\rho}_{j}\left|s_{j}\right|=-g s_{j}^{2} \leq 0$

So the system is global stability.

The sliding robust compensation is:

$$
U_{j}^{s}=\rho_{j} \operatorname{sat}\left(s_{j} / \Phi_{j}\right)
$$

D. The Configuration of the dynamic inversion adaptive sliding mode control

The configuration of the dynamic inversion adaptive sliding mode controller is designed as Figure 1.

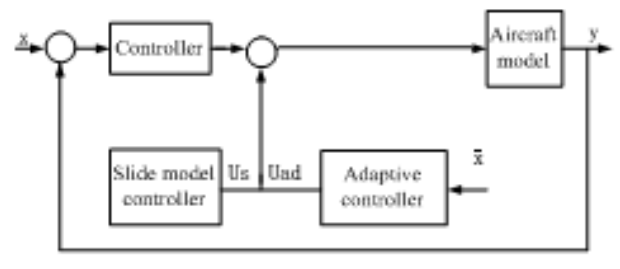

Figure 1 The configuration of controller

\section{SimUlation AND Results}

The initial flight state is $\mathrm{H}=3000 \mathrm{~m}$, Mach=0.4. The Figure 2 is pitch angle response curve. The simulation result shows that the dynamic inversion adaptive sliding mode flight controller has good control precision. The Figure 3 is pitch angle response curve in the condition of $+50 \%$ model perturbance. The solid line represents the dynamic inversion adaptive sliding mode flight controller. The dashed line represents the classical method. Comparing with the classical method, dynamic inversion adaptive sliding mode flight controller has better control performance.

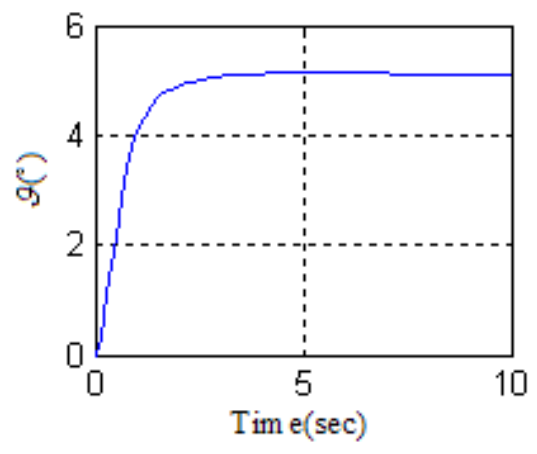

Figure 2. Pitch angle response

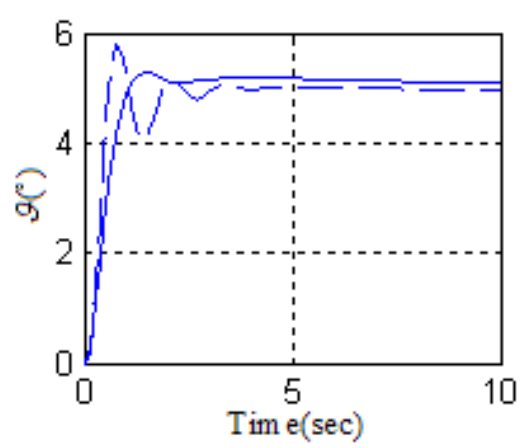

Figure 3. Pitch angle response(+50\% model perturbance)

\section{CONCLUSION}

A dynamic inversion adaptive sliding mode flight control method is approved in this paper. First, the drogue 
and 6-DOF refueling airplane model are built. Then, dynamic inversion method is used to realize the nonlinear control and fuzzy adaptive module is used to compensate the error of inversion. For improving the robustness, sliding mode control is used to optimize the adaptive control. The simulation results show: comparing with the classical method, this control method has higher control precision and excellent control performance.

\section{REFERENCES}

[1] Kim B S, Calise A J, Nonlinear Flight Control using Neural Networks, AIAA Journal of Guidance, Control and Dynamics(S0731-5090), 1997, 20(1): 26-33.

[2] Roshawn Elizabeth Bowers, Estimation Algorithm for Autonomous Aerial Refueling Using A Vision based Relative Navigation System, Texas A\&M University, 2005.

[3] Stepanyan Vahram, Lavretsky Eugene, Hovakimyan Naira. Aerial Refueling Autopilot Design Methodology Application to F-16 Aircraft Model, AIAA Guidance, Navigation, and Control
Conference and Exhibit 16-19 August 2004, Providence, Rhode Island AIAA 2004-5321.

[4] Joseph P Nalepka, Jacob L Hinchman, Automated Aerial Refueling Extending the Effectiveness of UAVs, AIAA -2005-6005.

[5] Lorenzo Pollini, Mario Innocenti, Roberto Matr, Vision Algorithms for Formation Flight and Aerial Refueling with Optidal Marker Labeling, AIAA - 2005 - 60101

[6] Jacob Hinchman, Automated Aerial Refueling Research Summary Presentation, AFRL - VA - WP - TP - 2003 - 344.

[7] Wisek B J, "Stability and flying quality robustness of a dynamic inversion aircraft control law," J. of Guidance, Control and Dynamics(S0731-5090), 1996, 19(6):1270-1278

[8] Y.S.Lu, J.S.Chen, “A Self-organizing Fuzzy Sliding-mode Controller Design for a Class of Nonlinear Servo Systems," IEEE Trans. On Industrial Electronics, vol.41, no.5, pp.492-502, 1994.

[9] C.M.Lin, C.H.Chen, “Adaptive Recurrent Fuzzy Neural Network Control for Linearized Multivariable Systems,” IEEE International Conf o Fuzzy Systems, 2004.

[10] Kimmett J, Valased J , Junkins J, Autonomous Aerial Refueling Utilizing A Vision Based Navigation System, AIAA - 2002- 4469. 